\title{
Journal of Hematology \& Oncology reviewer acknowledgement 2014
}

Delong Liu ${ }^{1}$ and Thalyana Smith-Vikos ${ }^{2^{*}}$

\section{Contributing reviewers}

The editors of Journal of Hematology \& Oncology would like to thank all our reviewers who have contributed to the journal in Volume 7 (2014).

\author{
Akintunde Akinleye \\ United States of America \\ Ramon Alemany \\ Spain \\ Veerajalandhar Allareddy \\ United States of America \\ Lyndal Anderson
Australia \\ Peter Anderson \\ United States of America \\ Shunya Arari \\ Japan \\ Diego Arenas \\ Mexico \\ Tomohiro Asai \\ Japan \\ Michele Baccarani \\ Italy \\ Jonathan Back \\ Switzerland \\ Elisabetta Baldi \\ Italy \\ Frederic Baron \\ Belgium
}

David Bartlett
United States of America

Amer Beitinjaneh

United States of America

Leonidas Benetatos

Greece

Jackie Boultwood

United Kingdom

M Mitzi Brentani

Brazil

Hal Broxmeyer

United States of America

Davide Calebiro

Germany

Dan Canaani

Israel

Daniel Catovsky

United Kingdom

Angela Chan

Canada

Hong Chang

Canada

Andy Chase

United Kingdom
Guoan Chen

United States of America

Michelle Y.Q. Chen

China

Yi-Bin Chen

United States of America

Sreenivasulu Chintala

United States of America

William CS Cho

Hong Kong

David Chui

United States of America

Monika Csóka

Hungary

Zoran Culig

Austria

Nico Dantuma

Sweden

Naval Daver

United States of America

Guido David

Belgium

Marc De Braekeleer

France

\footnotetext{
* Correspondence: jhoonline@biomedcentral.com

BioMed Central, Floor 6, 236 Gray's Inn Road, London WC1X 8HB, UK

'Department of Medicine, New York Medical College and Westchester

Medical Center, Valhalla 10595, NY, USA
} 


\begin{tabular}{|c|c|c|}
\hline Silvia Deaglio & Alvaro Gonzalez & Tomoo Iwakuma \\
\hline Italy & Spain & United States of America \\
\hline Jiusheng Deng & Finn Grey & Yongping Jiang \\
\hline United States of America & United Kingdom & United States of America \\
\hline Husheng Ding & Manuel Grez & Zhong Jiang \\
\hline United States of America & Germany & United States of America \\
\hline Kenneth Dorshkind & John Gribben & Zhenyu Ju \\
\hline United States of America & United Kingdom & China \\
\hline Sinisa Dovat & Aihua Gu & Hsueh-Fen Juan \\
\hline United States of America & China & Taiwan \\
\hline Madeleine Duvic & Chunhua Guo & Tamar Juven-Gershon \\
\hline United States of America & United States of America & Israel \\
\hline Brian Dymock & Hans Haecker & Dieter Kabelitz \\
\hline Singapore & United States of America & Germany \\
\hline Agnieszka Dzikiewicz-Krawczyk & James Hagman & Bin Kang \\
\hline Poland & United States of America & United States of America \\
\hline Andreas Eisenreich & Hideo Harigae & Lyndal Kearney \\
\hline Germany & Japan & United Kingdom \\
\hline Monica Else & Claire Harrison & Sophia Khaldoyanidi \\
\hline United Kingdom & United Kingdom & United States of America \\
\hline Monika Engelhardt & Dongmei He & Evgeny Klyuchnikov \\
\hline Germany & China & Germany \\
\hline Ferry ALM Eskens & Jeff Holst & Sergej Konoplev \\
\hline Netherlands & Australia & United States of America \\
\hline Lucia Farina & Michael Hsiao & Gopal Kundu \\
\hline Italy & Taiwan & India \\
\hline Jay Fox & Chung-Tsen Hsueh & Ann LaCasce \\
\hline United States of America & United States of America & United States of America \\
\hline Ota Fuchs & He Huang & Stephen Langabeer \\
\hline Czech Republic & China & Ireland \\
\hline Hiroshi Fukamachi & Xiaojun Huang & Alessandro Laviano \\
\hline Japan & China & Italy \\
\hline Muhammad Furqan & Suming Huang & Hillard Lazarus \\
\hline United States of America & United States of America & United States of America \\
\hline Shou-Jiang Gao & Kam HUI & John Lednicky \\
\hline United States of America & Singapore & United States of America \\
\hline Enrico Garattini & Jung-Jyh Hung & Claudia Lengerke \\
\hline Italy & Taiwan & Switzerland \\
\hline Valter Gattei & Motoshi Ichikawa & Sirpa Leppä \\
\hline Italy & Japan & Finland \\
\hline Christian Gisselbrecht & Shinsuke Iida & Vera Levina \\
\hline France & Japan & United States of America \\
\hline Damjan Glavac & Michael Ittmann & Chengang LI \\
\hline Slovenia & United States of America & United States of America \\
\hline
\end{tabular}


Qiang Li

United States of America

Shaoguang Li

United States of America

Yangqiu Li

China

Patrick Lin

United States of America

Ke Liu

United States of America

Wenyu Liu

United States of America

Jun Liu

United States of America

Pengyuan Liu

United States of America

Luca Lo Nigro

Italy

Olli Lohi

Finland

Xiaolin Luo

United States of America

Nigel Mackman

United States of America

Daichi Maeda

Japan

Saradhi Mallampati

United States of America

Sridhar Mani

United States of America

Francesco Mannelli

Italy

John Mascarenhas

United States of America

Kunio Matsumoto

Japan

Hiromichi Matsushita

Japan

Anna Rita Migliaccio

United States of America

Ken Mills

United Kingdom

Chang-Ki Min

South Korea
Zdravko Mitrovic

Croatia

Yohei Miyagi

Japan

Helmout Modjtahedi

United Kingdom

L. Rhoda Molife

United Kingdom

Andrei Moroz

Brazil

Alison Moskowitz

United States of America

Walid Mourad

Canada

Marek Mraz

Czech Republic

Hasan Mukhtar

United States of America

Marta Muzio
Italy

Hideaki Nakajima

Japan

Ryotaro Nakamura

United States of America

David Naor

Israel

Javier Naval

Spain

Jiri Neuzil

Australia

Siok Bian Ng

Singapore

Francesco Novelli

Italy

Seiichi Okabe

Japan

Lucy Osborne

Canada

Thomas Pabst

Switzerland

Chong-xian Pan

United States of America

Samir Parekh

United States of America
Jino Park

United States of America

William Parker

United States of America

Louis Pelus

United States of America

Archibald Perkins

United States of America

Iver Petersen

Germany

Jan Peveling-Oberhag

Germany

Linda Pilarski

Canada

Charalampos Pontikoglou

Greece

Louise Purton

Australia

Raju Pusapati

United States of America

Karen Rabin

United States of America

John Radford

United Kingdom

Joseph Ragaz

Canada

VK Rajasekhar

United States of America

Pavan Mahendra Ravella

United States of America

Ruibao Ren

United States of America

Hugh Reyburn

Spain

Adam Rich

United States of America

Ingo Ringshausen

United Kingdom

Marthe Rizk-Rabin

France

Maria Rizzo

Italy

Pierre Rohrlich

France 
Luisa Romao

Portugal

Emanuela Rosati

Italy

Stefan Rose-John

Germany

Patricia Rousselle

France

Jeffrey Rubnitz

United States of America

Ahmad Safa

United States of America

Kuniaki Saito

Japan

Ralph Salloum

United States of America

Brenda Sandmaier

United States of America

Bipin Savani

United States of America

Peter Sayeski

United States of America

Tobias Schatton

United States of America

Udo Schumacher

Germany

Heidi Schwarzenbach

Germany

Karen Seiter

United States of America

Carmine Selleri

Italy

Xiaojin Sha

United States of America

Bin Shan

United States of America

Mala Shanmugam

United States of America

Yukari Shirasugi

Japan

Yongqian Shu

China

Mitchell Smith

United States of America
Wenru Song

United States of America

Michael Spinella

United States of America

Eva Szegezdi

Ireland

Shinichiro Takahashi

Japan

Fumi Takahashi-Yanaga

Japan

Kazuhiro Tamura

Japan

Corrado Tarella

Italy

Pierfrancesco Tassone

Italy

Ugo Testa

Italy

Kensei Tobinai

Japan

Sanne Tonino

Netherlands

Jorg Tost

France

Ivo Touw

Netherlands

Mounir Trimeche

Tunisia

Volodymyr Tryndyak

United States of America

William Tse

United States of America

David Tulasne

France

Jeffrey Tyner

United States of America

Susumu Uchiyama

Japan

Anke van den Berg

Netherlands

Rosa Visone

Italy

Lydia Visser

Netherlands
Donghai Wang

United States of America

Kankan Wang

China

Qinhong Wang

United States of America

Yu Wang

United States of America

Erik Wiemer

Netherlands

Justin Wong

Australia

Bas Wouters

Netherlands

Kongming Wu

China

Jennifer Wu

United States of America

Yan Wu

United States of America

Dazhong Xu

United States of America

Hui Xu

United States of America

Mingjiang Xu

United States of America

Xiang-Xi Xu

United States of America

\section{Zhen XU}

United States of America

Xiao-Feng Yang

United States of America

Jilong Yang

China

Sherry Yang

United States of America

B. Hilda Ye

United States of America

Go Yoshida

Japan

M. James You

United States of America

Nadia Zaffaroni

Italy 
Apostolos Zaravinos

Sweden

Ali Zarrin

United States of America

Haiyan Zhai

United States of America

Qing Zhang

China

Suping Zhang

United States of America

Hua Zhang

China
Mingjun Zhang

United States of America

Dekuang Zhao

United States of America

Meng Zhao

United States of America

Xiaohong Zhao

United States of America

Xiaohong Zheng

United States of America

Minghao Zhong

United States of America
Liang Zhou

United States of America

Yong Zhou

China

Pier Luigi Zinzani

Italy

Vassilis Zoumpourlis

Greece 